\title{
Sistema automatizado para la elaboración de la característica tractiva en las máquinas automotrices. Tractiva 4.1
}

Software to tractive effort characteristic in ground vehicles. Tractiva 4.1

\author{
Esteban López Miláñ ${ }^{1}$ Erik Reyes Gómez ${ }^{1}$ y Buenaventura Rigol Cardona ${ }^{1}$
}

\section{RESUMEN}

La característica tractiva se calcula con el fin de mostrar las cualidades del movimiento de los vehículos automotores y es única, en correspondencia con el modelo del motor y el sistema de transmisión de fuerzas. Al realizar alguna modificación en estos elementos cambia también la respuesta cinemática y dinámica del vehículo y con ello: su característica tractiva. En este trabajo se muestra un sistema automatizado, diseñado en Visual Basic 6, que permite la elaboración de esta característica para cualquiera sea el vehículo del cual se dispongan los datos necesarios. El sistema interactúa a su vez con una base de datos de motores de automóviles y de datos técnicos de vehículos que facilitan su manejo. Los investigadores interesados en diseñar o mejorar las prestaciones de los automóviles, tienen en este sistema automatizado una útil herramienta de trabajo, que en tiempo récord les permitirá evaluar diferentes automóviles o valorar posibles modificaciones a éstos.

Palabras clave: característica tractiva; dinámica; automóviles.

\begin{abstract}
ABSTACT
The tractive effort characteristic shows the vehicle performance; it is unique in correspondence with the engine and power transmission of vehicle. Each transforming in this scheme causes a new response dynamic and kinematic of vehicle, such as it tractive effort characteristic. This paper shows a software, making in Phyton QT, to do the tractive effort characteristic for any vehicle. The software access to a database of engines and technical data of vehicles. The researchers that are interesting in design or improve the performance of ground vehicles, has in this software a good tool to evaluate different automobiles or to tests possible changes in design stage.
\end{abstract}

Keywords: tractive effort characteristic; automobile; dynamic.

\footnotetext{
1 Universidad de Holguín. Cuba.
} 


\section{ICHIKLLACHAW}

Traktiwa nishqan kanqantaqa musyatsimantsik imanawmi llapan kaarukunapa alli kuyunin kanqantam, muturwan kallpanpa kuyuy witsiyninqa manam kantsu. Kaykunachaw kaarukunapa tikrayninwanqa imallamanpis sinimatikawan dinamikanqa paqwaymi tikrarin, niykurpis qatillaman traktiwa nishqanpis patsaypam tikrarin. Kay uryaychawqa awtumatisadu nishqanmi rikakaarin, tsaymi Visual Basic 6 nishqanwan rurashqa. Kaymi yanapan imanaw rikuq kaaru rurakaananpaqpis. Kay muturwan tikniku kuyuykuyninkunaqa tsay daatu nishqanwanmi kuyun, tsayran pushanapaqpis alli. Musyapakuq yachaqkunaqa awtukuna alli ruraynichaw tsay awtumatisadu nishqan sistimankunam kayaapun allilla uryaykunachaw yarqunanpaq, tsay nishqantsiktaqa patsa aywayninchawmi alli kayninta tsanintsaatsiktsik.

Pushaq shimikuna: traktiwa imanaw kanqan; awtukunapa; kuyukuynin.

\section{INTRODUCCIÓN}

El fabricante de los automóviles proporciona el pasaporte dinámico (o característica tactiva) conjuntamente con la entrega de una unidad nueva, pero este pasaporte resulta inútil con cada modificación que se le realice al automóvil, puesto que entonces se verifica en la máquina una nueva respuesta dinámica y cinemática ante iguales condiciones de explotación. Por esta razón es conveniente antes de realizar modificaciones en el conjunto motor-sistema de transmisión de fuerzas, calcular la nueva característica tractiva y determinar con ello, si se cumplirán las expectativas para las cuales se desea llevar a cabo la modificación.

La característica tractiva se calcula con el fin de mostrar las cualidades del movimiento del automóvil y es la representación gráfica de la ecuación general de su movimiento. La ecuación general del movimiento del automóvil se obtiene, a su vez, a partir de la ecuación del balance de fuerzas sobre el vehículo.

$P n-W p-W d-P b-P u=0$

Entre estas fuerzas se destaca la fuerza tractiva $(P n)$ que provoca el movimiento de avance del automóvil y las restantes fuerzas que se oponen al movimiento del vehículo como son: la fuerza del viento o aerodinámica $(W p)$, la fuerza de resistencia del camino $(W d=W w+W t)$ compuesta por la resistencia a la rodadura $(W w)$ y la que causa la inclinación del camino $(W t)$, la fuerza en el gancho $(P u)$ y la fuerza de inercia $(P b)$.

Existen varias formas de representar la característica tractiva, aunque todas tienen en común la representación del comportamiento de la fuerza tractiva en función de la velocidad ( $V$ ). Como no se ha descrito una ley general que permita expresar la fuerza tractiva en función de la velocidad, el trazado de las curvas $P n(V)$ se consigue a través de ecuaciones paramétricas que permiten establecer pares coordenados $(\Psi, \Phi)$ de velocidad y fuerza tractiva. 


\section{MATERIALES Y MÉTODOS}

Este trabajo muestra un sistema automatizado que permite la elaboración de la característica tractiva, tomando lo esencial de la metodología establecida por López (2008) con similar fin. El sistema automatizado Tractiva 4.1, tiene su predecesor en el sistema Tractiva 2.1 (López y Senfort, 2001) y Tractiva 2.2 (López, 2009) el cual ha sido ampliamente usado por estudiantes de pregrado y posgrado de la carrera de Ingeniería Mecánica de la Universidad de Holguín.

La metodología en el sistema automatizado cuenta con los siguientes pasos:

1. Elaboración de la característica exterior de velocidad del motor.

Frecuentemente no se dispone de la característica exterior de velocidad del motor, pero siempre se puede contar con datos tales como la potencia nominal y las velocidades de rotación mínima, nominal y máxima del motor; estos datos resultan suficientes para calcular los valores de potencia y dar en las diferentes velocidades de rotación del motor.

2. Construcción de la tabla de velocidad $(V)$, resistencia del viento $(W p)$, fuerza tractiva $(P n)$ del automóvil y determinación de la eficiencia de la transmisión $\left(\boldsymbol{\eta}_{0}\right)$.

La velocidad de traslación de la máquina se calcula teniendo en cuenta la velocidad de rotación de sus neumáticos, el radio dinámico de las ruedas, la velocidad angular del motor y del valor de la relación de transmisión total del sistema de transmisión de fuerzas (Aragón, 1985), (Cárdenas, 1986), (Szczepaniak y Aragón, 1992).

3. Elección de la escala de fuerza tractiva y el factor dinámico.

La fuerza tractiva se escala en el eje de las ordenadas. Para establecer la altura del eje de fuerza tractiva, el área de trazado se divide aproximadamente a la mitad por la vertical y en esta línea divisoria, se ubica el eje de velocidad. En la parte superior del área dividida, se sitúa el cuadrante donde se traza el eje de fuerza tractiva y en la inferior, se establece la zona de la fuerza de resistencia del viento y la parte cinemática de la característica tractiva. A la izquierda de la escala de fuerza tractiva, se traza la escala del factor dinámico, el cual es directamente proporcional a la fuerza tractiva.

\section{Elegir la escala de velocidad.}

Los pasos para confeccionar la escala de velocidad (V), son similares a los establecidos para la escala de fuerza tractiva; pero a diferencia de lo anterior, los valores notables de velocidad se establecen en 10 ó $20 \mathrm{~km} / \mathrm{h}$ y el mayor valor del eje de velocidad no lo constituye el valor notable inmediatamente superior a la velocidad máxima (teórica), sino el propio valor de velocidad máxima. 
5. Trazado de los ejes de coordenadas.

6. Ubicación de los pares coordenados de $P n$ y $V$.

En la literatura consultada, no se ha encontrado una ecuación que permita establecer valores de fuerza tractiva en función de la velocidad del movimiento del automóvil; no obstante, sí es posible parear valores correspondientes de estas magnitudes, ya que están estrechamente relacionados.

7. Trazado de la resistencia del viento.

La resistencia del viento se traza usando la misma escala que la fuerza tractiva, este eje tiene igual dirección y sentido opuesto que la fuerza tractiva, partiendo del origen de coordenadas.

8. Trazado de la resistencia del camino.

El desplazamiento de los equipos automotores sobre la vía, implica el surgimiento de fuerzas que se oponen a su movimiento; estas fuerzas dependen del tipo, de las características y de la inclinación del camino. La resistencia del camino, se determina por la suma de las resistencias al movimiento de la máquina causadas por la resistencia al rodamiento y por la inclinación del camino.

9. Cálculo de la pendiente máxima y trazado.

De la ecuación general adimensional del movimiento del automóvil se conoce que la máxima pendiente que puede vencer el vehículo se alcanza en la primera marcha, cuando se obtiene la mayor de la fuerza tractiva posible; en esta marcha la fuerza de resistencia del viento es despreciable, el vehículo transita por condiciones óptimas de camino y se supone que el vehículo no tiene remolque alguno.

10. Cálculo de la fuerza de inercia y las aceleraciones.

La fuerza de inercia actúa sobre el automóvil cuando el movimiento del mismo no es uniforme. A menudo la fuerza de inercia es llamada resistencia debido a la inercia y se trata como una de las componentes de todas las resistencias que actúan sobre el automóvil durante su movimiento.

\section{Trazado de las aceleraciones.}

En la ecuación del balance de fuerzas se plantea que la sumatoria de fuerzas que intervienen durante el movimiento del automóvil es igual a cero. La condición esencial es que, para cada una de las marchas, se realiza el cálculo de la aceleración con la máxima fuerza tractiva y las mejores condiciones del camino (valores más bajos del coeficiente de resistencia a la rodadura). 


\section{Trazado de la parte cinemática.}

La parte cinemática tiene la función de mostrar las cualidades cinemáticas del vehículo, por cuanto relaciona la velocidad del movimiento de la máquina con las relaciones de transmisión conectadas en la caja de velocidades.

Como se aprecia, la elaboración de la característica tractiva es sumamente laboriosa, de aquí la necesidad de automatizar los cálculos.

El diseño del software se hizo siguiendo el patrón Modelo Vista Controlador, para facilitar una mejor interpretación del código y con el objetivo de contribuir a futuras mejoras del producto.

El programa está compuesto por cinco clases de datos, 16 clases controladoras y 16 clases interfaz. Igualmente cuenta con una base de datos relacional, la cual fue desarrollada utilizando Sqlite 3, compuesta por cuatro tablas que interrelacionan entre ellas y en las cuales se almacena los datos de los proyectos, automóviles, motores y neumáticos con los que opera el sistema. La figura 1 resume la estructura del sistema Tractiva 4.1.

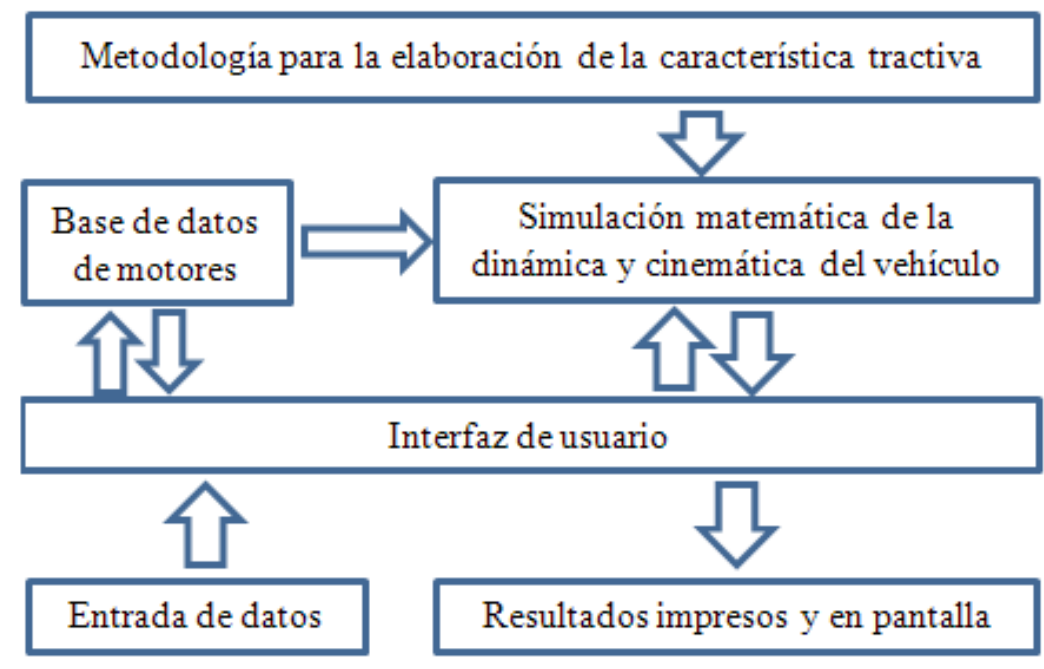

Figura 1. Estructura del sistema automatizado Tractiva 4.1

\section{RESULTADOS}

Esta nueva versión del sistema automatizado permite más libertades al usuario de cara al diseño del automóvil. El usuario decide respecto a las velocidades de movimiento del vehículo, tipo de camino al cual se destina el vehículo y características del neumático que se usa, entre otras cuestiones importantes.

Con la nueva concepción de este sistema es posible calcular las relaciones de transmisión en los elementos del sistema de transmisión de fuerzas y como las versiones anteriores, permite la elaboración del pasaporte dinámico de la máquina. 
Con el sistema Tractiva V4.1, pueden ser realizadas diversas operaciones tales como:

- Construcción de la gráfica de la característica tractiva.

- Consulta de valores de coeficientes (coeficiente de resistencia a la rodadura, coeficiente de adherencia y coeficiente aerodinámico).

- Acceso total a las bases de datos de motores y vehículos, permite modificar y añadir nuevos registros.

- El sistema calcula los parámetros cinemáticos de los elementos de la transmisión de fuerzas.

- Salva de datos de operaciones de cálculo y de imágenes en soporte digital.

- Análisis del movimiento de la máquina en condiciones límites.

- Carga de varias imágenes de características calculadas.

- Acceso a la calculadora del sistema operativo.

\section{DISCUSIÓN}

Al ejecutar Tractiva V4.1.exe, aparece una pantalla de inicialización con el nombre del producto, la cual contiene una imagen que representa el esquema de las fuerzas que intervienen durante el movimiento de los automóviles. Desde esta ventana principal se ejecuta todas las operaciones del sistema, las cuales están comprendidas en cuatro menús: Archivo, Componentes, Herramientas y Ayuda, como muestra la figura 2.

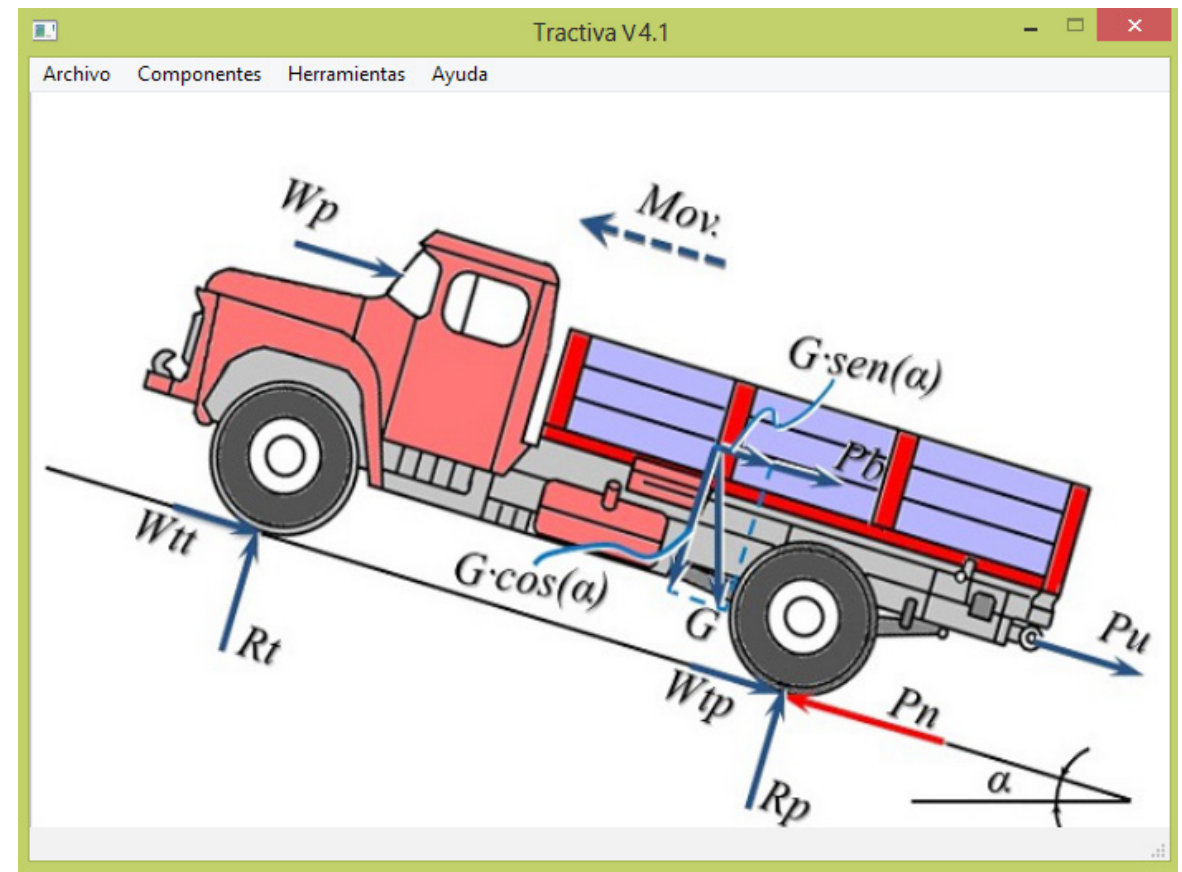

Figura 2. Formulario principal Tractiva 4.1 
Desde el menú «Archivo» se accede al comando para la ejecución de un nuevo proyecto, que da paso a un formulario en el cual se introducen datos a opción del usuario y/o accediendo a las bases de datos del sistema. La figura 3 muestra un caso de un nuevo proyecto formulado por el usuario.

\begin{tabular}{|c|c|c|c|}
\hline 国 & Nuevo Proyecto & $?$ & $\times$ \\
\hline Datos del Automóvil & Características del Motor & \multicolumn{2}{|c|}{ Características de los Neumáticos } \\
\hline Marca: KAMAZ & Marca: KAMAZ & \multicolumn{2}{|l|}{ Marca: 6} \\
\hline Modelo: 5200 & Modelo: KAMAZ -740 & \multicolumn{2}{|l|}{ Numeración: 7.00 D20 } \\
\hline Tipo de Vehículo: carga & Tipo: Diesel Cámara-Unica & \multicolumn{2}{|c|}{ Recomendado para: Indeterminado } \\
\hline Ancho $(\mathrm{mm}): 2500$ & Número de Cilíndros: 8 & \multicolumn{2}{|c|}{ Tipo de Neumático: Convencional } \\
\hline Alto $(\mathrm{mm}): 2630$ & Potencia Nominal: 154 & \multicolumn{2}{|l|}{ Ancho: 7.0} \\
\hline Peso del Vehículo Cargado (kg): 15300 & RPM Mín.: 700 & \multicolumn{2}{|l|}{ RING: 20} \\
\hline Peso del Remolque Cargado $(\mathrm{kg}): 0$ & RPM Nom.: 2600 & \multicolumn{2}{|l|}{ Radio Dinámico: 0.418} \\
\hline Coeficiente Aerodinámico: 0.95 & RPM Máx.: 2600 & & \\
\hline Transmisión: Sistema_Serie_tipo-2 & & & \\
\hline Eficiencia: 0.9 & & Cerr & \\
\hline
\end{tabular}

Figura 3. Formulario Datos del Automóvil

Desde el menú «Componentes» le es permitido al usuario la modificación de las bases de datos de automóviles, motores y neumáticos. Las acciones que puede realizar son las de edición de algún registro, adición de uno nuevo o sustracción de alguno existente, como se ejemplifica en la siguiente figura para el caso de los neumáticos.

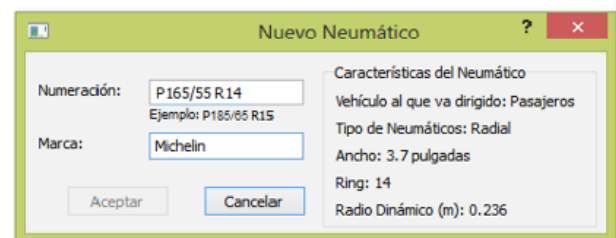

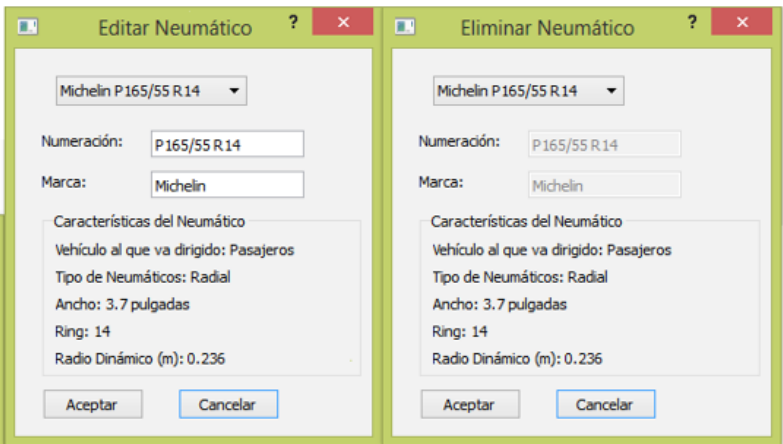

Figura 4. Formularios para el trabajo del componente «Neumático»

El menú «Herramienta» despliega las opciones para editar el proyecto, realizar el cálculo de la transmisión y graficar los resultados. 


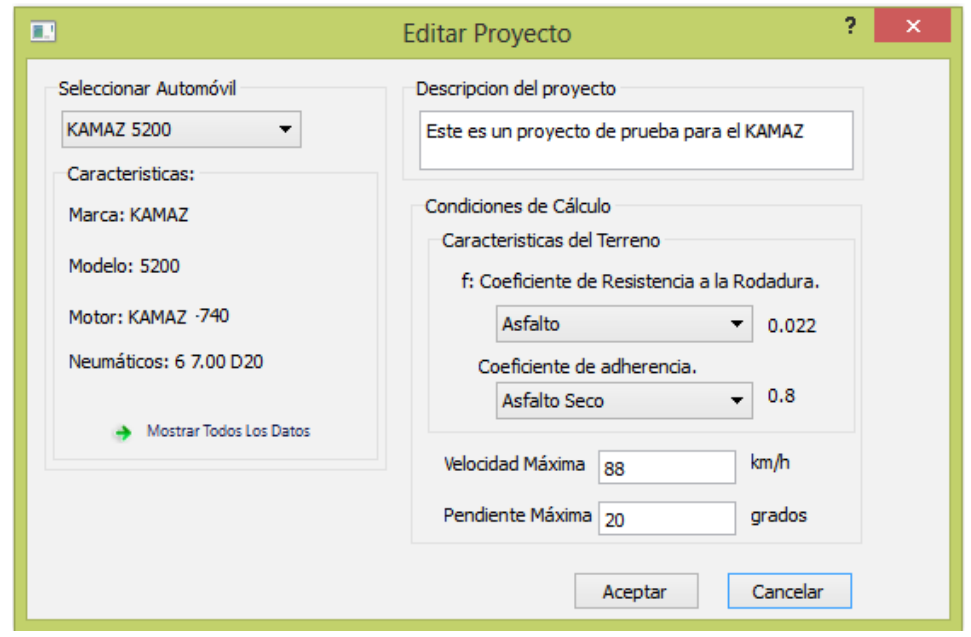

Figura 5. Formulario «Editar Proyecto»

El formulario «Editar Proyecto» tiene un diseño similar a «Nuevo Proyecto» del menú «Archivo», este tiene el objetivo de dar al usuario la oportunidad de editar el proyecto que ya se ha guardado y se necesita modificar sus datos para obtener nuevos valores en el cálculo.

El formulario «Cálculo de la Transmisión» (figura 6) al ser llamado por el usuario, calcula de manera automática la cantidad de marchas y las relaciones de transmisión, que necesita el automóvil para vencer las condiciones preestablecidas. El formulario también da la oportunidad de variar estas relaciones de transmisión, aprovechando la experiencia del usuario.

En el caso de que adicionalmente se tenga en el esquema de la transmisión una caja de reenvío o un divisor de velocidades, como resultado se duplica el número de marchas. Por defecto, en el formulario estas opciones aparecen con el valor uno y le da la posibilidad al usuario de modificar su valor. El usuario puede decidir también por una sobremarcha para el automóvil.

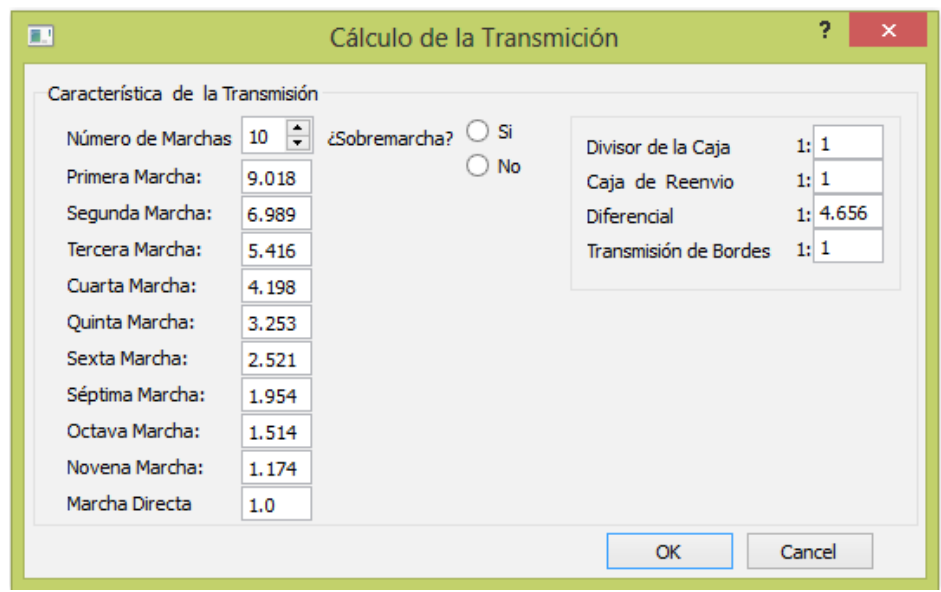

Figura 6. Formulario «Cálculo de la Transmisión» 
En el formulario «Graficar Resultados» (figura 7) se muestra la gráfica de la característica tractiva de un automóvil que posee diez marchas. En el eje vertical se establece la fuerza tractiva (en $\mathrm{kN}$ ), en el eje horizontal la velocidad (en $\mathrm{km} / \mathrm{h}$ ), en la parte superior del cuadrante se ha ubicado a la escala de la pendiente que se incrementa de derecha a izquierda.

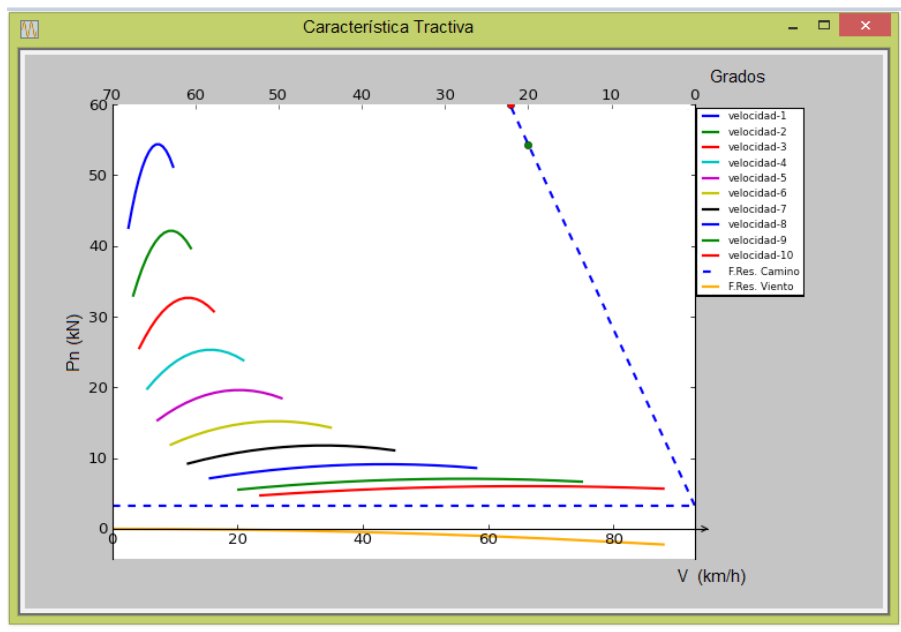

Figura 7. Gráfico de la característica tractiva de un automóvil

En la gráfica, se pueden apreciar las curvas que representan cada una de las marchas, la línea horizontal discontinua que señala el valor de la fuerza de resistencia del camino para una pendiente nula, y la discontinua inclinada que sirve para determinar las pendientes que puede vencer el automóvil en cada marcha y velocidad de movimiento.

En la parte inferior y con valores negativos, se traza la curva parabólica cúbica de fuerza de resistencia del viento que es entre otras cuestiones, función de la velocidad de movimiento del automóvil. Se completa la gráfica con una leyenda en la parte superior derecha con un código de colores para identificar las líneas de fuerza tractiva, resistencia aerodinámica y de resistencia del camino. A las curvas de fuerza tractiva que aparecen en la gráfica, previamente se les ha deducido el valor de la resistencia del viento a esa velocidad.

\section{CONCLUSIONES}

El disponer de una adecuada metodología para la elaboración de la característica tractiva y el cálculo de las relaciones de transmisión, ha sido parte fundamental en la solución al problema planteado, a partir de lo cual se ha llegado a la creación del sistema automatizado.

El sistema Tractiva 4.1, destinado a la elaboración de la característica tractiva de las máquinas automotrices, es un software que puede ser fácilmente asimilado por los especialistas en la dinámica y cinemática de las máquinas automotrices. En pocos minutos, 
básicamente en lo que dura la captura de los datos, es capaz de construir la característica tractiva de cualquier vehículo automotor a partir de sus parámetros originales o si es el caso, a partir de las modificaciones que se le introduzcan.

El software es capaz de evaluar y graficar el comportamiento de la máquina en la inmensa combinatoria de situaciones en que se puede desempeñar. Su uso ha estado dirigido fundamentalmente a la evaluación del comportamiento cinemático y dinámico del vehículo al cual se le pretende realizar alguna modificación, sea un cambio de tipo de motor, adaptación de otra caja de velocidades, de un diferencial, cambio de las dimensiones de los neumáticos o la combinación de estas posibilidades.

Este software (y su predecesor) ha demostrado su utilidad y confiabilidad de los resultados en diversas investigaciones realizadas, tanto en estudios de pregrado como en tesis de maestrías, especialidades y doctorados.

\section{REFERENCIAS BIBLIOGRÁFICAS}

Aragón, Rigoberto y Ortega, F. I. 1985. Manual del proyecto de curso de automóviles. Santiago de Cuba: Edit. ENSPES.

Cárdenas, Miguel. 1986. Prácticas de laboratorio de evaluación y prueba de vehículos. La Habana: Edit. ISPJAE.

López, Esteban. 2008. Contribuciones al perfeccionamiento del sistema de transporte cañero. Tesis doctoral. CENDA, La Habana.

López, Esteban y Méndez, Elizabeth. 2008. «La remotorización Diesel en los camiones ZIL 130. Un análisis técnico de factibilidad». Revista Ciencias Holguín. Vol. XIV, No 2.

López, Esteban. 2009. «Sistema «Tractiva 2.2» para la elaboración de la característica tractiva en las máquinas». Memorias de la IV Conferencia Científica Internacional Universidad de Holguín.

López, Esteban y Senfort, Julio. 2001. «Sistema TRACTIVA Versión 2.1». CENDA, La Habana. Registro de obra protegida: 07678-7678.

Szczepaniak, Cesary; Aragón, R. 1992. Teoría del automóvil. Edit. Pueblo y Educación. La Habana.

Fecha de recepción: 15 de junio de 2016

Fecha de aceptación: 20 de noviembre de 2016

\section{Correspondencia}

Esteban López Milán

elopez@facing.uho.edu.cu 